\title{
Evaluation of biocompatibility of novel and commonly-used antiseptics by cell culture method
}

\author{
M Yamamoto ${ }^{1 *}$, R Matsumura ${ }^{2}$, Y Hirata ${ }^{2}$, H Nagamune $^{1}$ \\ From 3rd International Conference on Prevention and Infection Control (ICPIC 2015) \\ Geneva, Switzerland. 16-19 June 2015
}

\begin{abstract}
Introduction
Antiseptic agents such as chlorhexidine digluconate, polyhexamethylene biguanide, benzalkonium chloride and octenidine dihydrochloride (octenidine) has been used widely in clinical settings to prevent bacterial infection, especially for antisepsis of skin and mucous membranes. However, irritation and cytotoxic effects of these compounds on human cell has been reported.
\end{abstract}

\section{Objectives}

This study compared the biocompatibility of newlysynthesized bis-quaternary ammonium compounds and biocides that have been commonly used as antiseptics, examining their antimicrobial activity, cytotoxic effect and expression of inflammatory cytokine-related genes.

\section{Methods}

A series of new bis-quaternary ammonium compounds, including 3-(3-Hydroxy-2-(hydroxymethyl)-2-\{[(1-dodecylpyridinium-3-yl)oxy]methyl\}propoxy)-1- dodecylpyridinium dibromide (3HHDMP) was synthesized.The antimicrobial activity of newly-synthesized compounds and commonlyused antiseptics were assessed by minimum bactericidal concentrations (MBC) and cytotoxic effect on normal human epidermal keratinocytes. The expression of inflammatory cytokines in normal human epidermal keratinocytes was quantified using real-time reverse transcription PCR (RT-PCR).

\section{Results}

Against Gram-negative and Gram-positive bacteria, 3HHDMP showed potent antimicrobial activity comparable to that of the octenidine. In a cytotoxic test using human epidermal keratinocytes, toxicity of 3HHDMP was equal or lower compared to that of quaternary ammonium compounds, although 3HHDMP showed higher toxicity than biguanide-based compounds. The comparison of the biocompatibility index as defined by antimicrobial activity and cytotoxic effect on human cell revealed that 3HHDMP had equal or greater biocompatibility compared with the biocides tested. Moreover, in the expression analysis of cytokine-related genes by cell culture method, increase of expression of cytokinerelated genes in the cells stimulated with 3HHDMP was slower than that of existing quaternary ammonium compounds.

\section{Conclusion}

From these results, it was concluded that the series of new bis-quaternary ammonium compounds including 3HHDMP had excellent biocompatibility, and thus the availability for application to the skin surface was suggested.

\section{Disclosure of interest}

None declared.

\section{Authors' details}

'Department of Biological Science and Technology, Institute of Technology and Science, Tokushima University Graduate School, Tokushima, Japan.

${ }^{2}$ Biochemical Laboratory, SARAYA CO. LTD., Osaka, Japan.

Published: 16 June 2015

doi:10.1186/2047-2994-4-S1-P38

Cite this article as: Yamamoto et al:: Evaluation of biocompatibility of novel and commonly-used antiseptics by cell culture method. Antimicrobial Resistance and Infection Control 2015 4(Suppl 1):P38. 